OPEN ACCESS

Edited by:

Mitsuo Tagaya,

Tokyo University of Pharmacy and Life

Sciences, Japan

Reviewed by:

Jyoti K. Jaiswal,

Children's National Hospital,

United States

*Correspondence:

Carlos Enrich

enrich@ub.edu

Specialty section:

This article was submitted to

Membrane Traffic,

a section of the journal

Frontiers in Cell and Developmental

Biology

Received: 19 October 2021 Accepted: 16 December 2021

Published: 06 January 2022

Citation:

Enrich C, LuA, TebarF, Rentero C and Grewal T (2022) Annexins Bridging the Gap: Novel Roles in Membrane

Contact Site Formation.

Front. Cell Dev. Biol. 9:797949.

doi: 10.3389/fcell.2021.797949

\section{Annexins Bridging the Gap: Novel Roles in Membrane Contact Site Formation}

\author{
Carlos Enrich $^{1,2 *}$, Albert Lu ${ }^{1,2}$, Francesc Tebar ${ }^{1,2}$, Carles Rentero ${ }^{1,2}$ and Thomas Grewal ${ }^{3}$ \\ ${ }^{1}$ Departament de Biomedicina, Unitat de Biologia Cel.lular, Facultat de Medicina i Ciències de la Salut, Universitat de Barcelona, \\ Barcelona, Spain, ${ }^{2}$ Centre de Recerca Biomèdica CELLEX, Institut d'Investigacions Biomèdiques August Pi i Sunyer (IDIBAPS), \\ Barcelona, Spain, ${ }^{3}$ School of Pharmacy, Faculty of Medicine and Health, University of Sydney, Sydney, NSW, Australia
}

Membrane contact sites (MCS) are specialized small areas of close apposition between two different organelles that have led researchers to reconsider the dogma of intercellular communication via vesicular trafficking. The latter is now being challenged by the discovery of lipid and ion transfer across MCS connecting adjacent organelles. These findings gave rise to a new concept that implicates cell compartments not to function as individual and isolated entities, but as a dynamic and regulated ensemble facilitating the trafficking of lipids, including cholesterol, and ions. Hence, MCS are now envisaged as metabolic platforms, crucial for cellular homeostasis. In this context, well-known as well as novel proteins were ascribed functions such as tethers, transporters, and scaffolds in MCS, or transient MCS companions with yet unknown functions. Intriguingly, we and others uncovered metabolic alterations in cell-based disease models that perturbed MCS size and numbers between coupled organelles such as endolysosomes, the endoplasmic reticulum, mitochondria, or lipid droplets. On the other hand, overexpression or deficiency of certain proteins in this narrow $10-30 \mathrm{~nm}$ membrane contact zone can enable MCS formation to either rescue compromised MCS function, or in certain disease settings trigger undesired metabolite transport. In this "Mini Review" we summarize recent findings regarding a subset of annexins and discuss their multiple roles to regulate MCS dynamics and functioning. Their contribution to novel pathways related to MCS biology will provide new insights relevant for a number of human diseases and offer opportunities to design innovative treatments in the future.

Keywords: annexins, membrane contact sites, endolysosomes, mitochondria, endoplasmic reticulum, cholesterol, calcium-binding proteins, lipid transport

\section{INTRODUCTION}

Despite the identification of membrane contacts between neighbouring organelles in the early times of electron microscopy, these small microdomains only received greater attention in the last decade. Two findings prompted further research on the structure and function of membrane contact sites (MCS). Firstly, the endoplasmic reticulum (ER) representing a dynamic 3D network of cisternae and tubules, it fills the cytoplasm and is in physical contact with other organelles (Nixon-Abell et al., 2016). Secondly, the discovery of the physiological relevance of contacts between the ER and mitochondria (mitochondria-associated membranes, MAMs) in the synthesis and exchange of lipids and calcium $\left(\mathrm{Ca}^{2+}\right)$ homeostasis (Vance, 1990). Since then, the MCS-related Universe expanded 
A

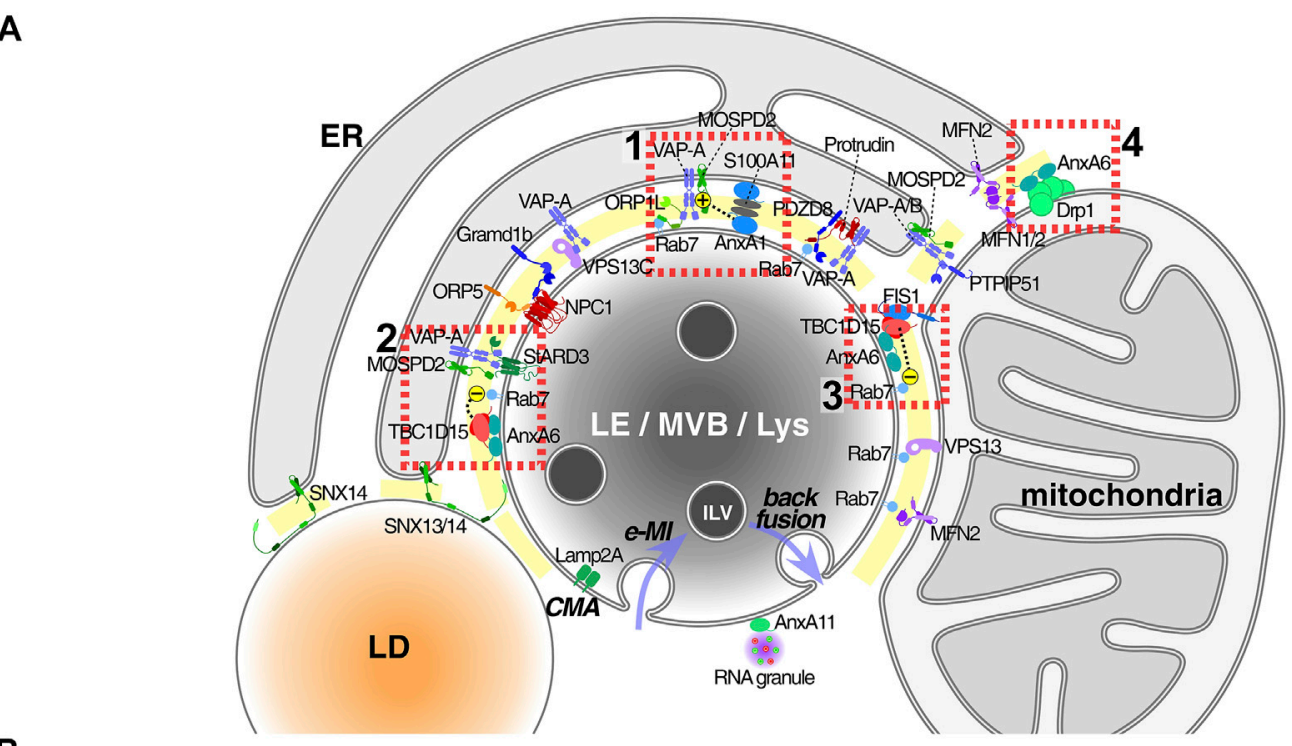

B

1

2

3

4

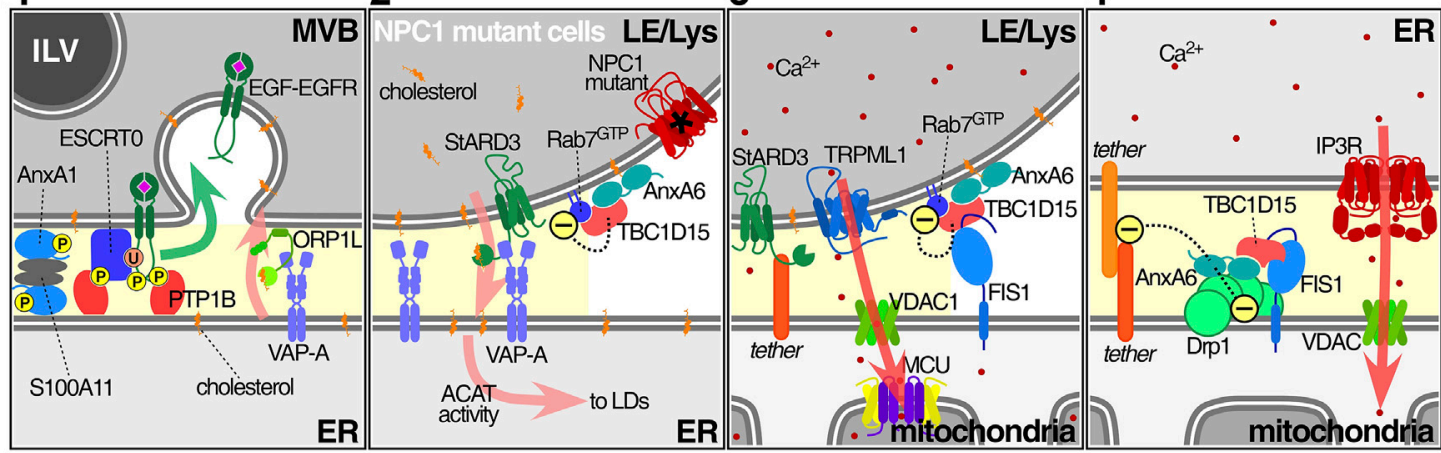

C

O KFEQ-motif constitutive

FIGURE 1 | Annexins and associated protein complexes at membrane contact sites. (A) Endolysosomes (LE/MVBs/Lys) establish multiple membrane contact sites (MCS) with a variety of other organelles, in particular the ER and mitochondria, but also peroxisomes and lipid droplets. Here we only illustrate a subset of contacts and contact site proteins. Lipid transfer, including cholesterol as well as $\mathrm{Ca}^{2+}$ mobilization and signalling are probably the most important MCS-associated functions. Annexins have been found in MCS connecting the ER with either LE or mitochondria (MAMs), as well as in LE-mitochondria contacts. Other proteins or protein complexes serve as tethers to ensure the formation of MCS, including several sorting nexins (Henne et al., 2015; Dong et al., 2016; Saric et al., 2021), VAP proteins, PTPIP51, PDZD8 or protrudin (Shirane et al., 2020; Neefjes and Cabukusta, 2021). Furthermore, ORP5/8 and NPC1/Gramd1 also translocate lipids including PS and cholesterol, across MCS between the ER and mitochondria and/or LE respectively (Galmes et al., 2016; Hoglinger et al., 2019). At the bottom of this endolysosome, we contemplate the possible recruitment of cytosolic annexins into ILV (see text for details) via two autophagic pathways. While chaperone-mediated and Lamp2adependent autophagy (CMA) may carry annexins into the lumen of MVBs or the outer membrane of ILVs, endosomal microautophagy (e-MI) may board annexins inside ILV (both routes via KFERQ-motif) (Tekirdag and Cuervo, 2018). In any case, whatever the topology, these proteins have to escape from lysosomal degradation (Meneses-Salas et al., 2020b). Then, ILV might undergo back fusion/retrofusion (Eden and Futter, 2021), delivering annexins and/or other cargo out of the MVB into the cytosol in the vicinity of MCS, where a suitable local $\mathrm{Ca}^{2+}$ and lipid microenvironment could then favour retention in MCS. Alternatively, MVB diversion to exocytosis could generate exosomes. This could be the destination of AnxA11, highly enriched in exosomes. Otherwise, cytosolic AnxA11 could be confined to PI(3,5)P2 at the endolysosomal cytosolic membrane to tether RNA granules (Liao et al., 2019). (B) 1: AnxA1, together with S100-A11 as a tetrameric complex, tethers MCS to mediate cholesterol transport from ER to LE/Lys via interaction of VAP-A with ORP1L (Eden et al., 2016). In this scenario, AnxA1 overexpression increases MCS between MVB and ER (Wong L. H. et al., 2018). 2: AnxA6 overexpression decreased MCS numbers between ER and LE/Lys, whereas AnxA6 depletion in NPC1 mutant cells stimulated MCS formation. The underlying mechanism involves the recruitment of AnXA6 and TBC1D15 to Rab7-positive organelles and was associated with increased

(Continued) 
FIGURE 1 | LE motility and LE-cholesterol release into the ER, through StARD3-VAP-A in MCS (Meneses-Salas et al., 2020b). 3: TBC1D15 and Rab7 in complex with FIS1 between LE/Lys and mitochondria affects the fission of mitochondria (Wong Y. C. et al., 2018). 4: AnxA6 interacts with Drp1 and FIS1 between ER and mitochondria to modulate $\mathrm{Ca}^{2+}$ dynamics and mitochondrial fission (Chlystun et al., 2013). In all settings shown in Insets 2-4 the presence of AnxA6 seems to cause MCS untethering. Arrows indicate the following: translocation of EGFR-EGF into ILV (green, inset 1), cholesterol flux (pink, insets 1-2), Ca ${ }^{2+}$ flux (red, insets 3-4). (C) Schematic representation of the domain structure of the three annexins found in MCS: Motifs that may be involved in the recruitment annexins to MCS are indicated and include the homology to FFAT motifs (blue), S100-binding sites (green) (Rety et al., 2000; Chang et al., 2007; Rintala-Dempsey et al., 2008) and KFERQ-motifs (yellow and red) (Cuervo et al., 2000). Abbreviations that do not appear in the text: ACAT, Acyl-CoA:cholesterol acyltransferase; MCU, mitochondrial calcium uniporter; FIS1, mitochondrial fission 1; IP3R, inositol 1,4,5-triphosphate receptor; TRPML1, transient receptor potential mucolipin 1; Vps13, Vacuolar protein sorting-associated protein 13; MFN1/2, mitofusin1/2; Gramd1b, GRAM domain containing 1B; VAP proteins, VAP-A, VAP-B; monomer specific d-peptide 1 (MOSD1, 2 and 3) and PS, phosphatidylserine.

rapidly and MCS are now considered metabolic platforms for the transport of small molecules such as lipids and ions (Prinz et al., 2020). In addition, MCS modulate various other functions, including organelle trafficking, endosome maturation and positioning, membrane dynamics, $\mathrm{Ca}^{2+}$ signalling, autophagy and membrane/vesicle/organelle fusion and fission (Friedman et al., 2013; van der Kant and Neefjes, 2014; Raiborg et al., 2015a; Raiborg et al., 2015b; Eisenberg-Bord et al., 2016; Abrisch et al., 2020; Silva et al., 2020). Membrane contacts also exist in organelles with internal membranes, including mitochondria, chloroplasts, and multivesicular bodies (MVBs) (Prinz et al., 2020). As MCS formation can be manipulated experimentally and is de-regulated in human disease models (Wu et al., 2018; Henne, 2019; Scorrano et al., 2019; Ballabio and Bonifacino, 2020; Martello et al., 2020; Petkovic et al., 2021), MCS have become attractive therapeutic targets.

MCS appear as small contact zones between neighbouring organelles, but contain a plethora of proteins and lipids. The biogenesis, maintenance, dynamics, and function of MCS rely on protein-protein interactions bridging apposed membranes to establish their communication. These proteins in MCS include numerous lipid and cholesterol transfer proteins, and a variety of tethers, sorting nexins, membrane channels, SNAREs, small RabGTPases and their regulators. In addition, as outlined in more detail below, several annexins are also found in MCS. It would go beyond the scope of this Mini Review to list all proteins and we recommend excellent articles and reviews on this topic (Alpy and Tomasetto, 2005; Eisenberg-Bord et al., 2016; Kentala et al., 2016; Atakpa et al., 2018; Hoyer et al., 2018; Kumar et al., 2018; Quon et al., 2018; Sandhu et al., 2018; Balla et al., 2019; Bohnert, 2019; Liao et al., 2019; Patel, 2019; Cremer et al., 2020; Islinger et al., 2020; Meneses-Salas et al., 2020b; Peng et al., 2020; Hewlett et al., 2021; Reinisch and Prinz, 2021; Saric et al., 2021; Wu and Voeltz, 2021).

\section{Annexins-Novel Players in Intracellular Communication and Membrane Contact Sites}

Annexins are characterized by a high structural homology that enables binding to acidic phospholipids in a $\mathrm{Ca}^{2+}$-dependent manner. In fact, most annexin functions in cells are due to their dynamic and reversible binding to membranes (Gerke et al., 2005; Enrich et al., 2017). More recently, mouse models lacking individual annexins validated proposed functions in complex physiological processes in vivo (Alvarez-Guaita et al., 2020;
Grewal et al., 2021). Annexins are present in multiple cellular compartments to regulate numerous functions, including membrane trafficking, cytoskeleton dynamics, ion channels, cell signalling, membrane repair and pro- or antiinflammatory activities. Most relevant for this review, annexins (AnxA1, A6 and A11) are located in endolysosomes in the vicinity of the ER (Figure 1A) with novel MCS-related roles for these annexins in cell physiology (Pons et al., 2000; Eden et al., 2016; Liao et al., 2019).

AnxA1 is ubiquitously expressed and found at the plasma membrane, endo-/exocytic vesicles, the cytoskeleton and nucleus (Grewal et al., 2021). Moreover, AnxA1 acts as a tether connecting the ER and MVBs (Eden et al., 2010; Eden et al., 2016). Tyrosine phosphorylated AnxA1 together with its binding partner S100A11 provide a docking site for tyrosine phosphatase 1B (PTP1B), an enzyme localized in the ER, at MVB-ER contacts to enable sorting of ligand-stimulated epidermal growth factor receptor (EGFR) onto intraluminal vesicles (ILVs) (Figure 1B1). EGF-induced AnxA1 phosphorylation is crucial for the segregation of EGFR onto ILVs (White et al., 2006). It remains to be clarified if AnxA1 acts in concert with the ESCRT complex and associated proteins and lipids (Raiborg and Stenmark, 2009) to facilitate this indispensable step targeting EGFR to lysosomes for degradation. Thus, MVB-ER contacts may provide localized sites where the phosphorylation status of AnxA1 and MVB sorting machinery components could be tightly controlled (Futter et al., 1993; White et al., 2006; Du et al., 2012; Eden et al., 2016).

Importantly, the AnxA1 tethering function is also required for ER-derived cholesterol transport to MVBs, a critical step for ILV formation to spatially regulate EGFR signalling. When cholesterol levels are low due to reduced endocytosis of Low-density lipoproteins (LDL), AnxA1-regulated membrane contacts facilitate cholesterol transfer from the ER to MVBs via the interaction of ER-localized VAMP-associated proteins (VAPs) and the endosomal oxysterol-binding protein related protein $1 \mathrm{~L}$ (ORP1L) (Eden et al., 2016).

Besides the AnxA1-S100A11 complex, other annexins also interact with S100 proteins, and may interact with two membranes simultaneously (Rescher and Gerke, 2004; RintalaDempsey et al., 2008). Hence, the reversible membrane binding capacity of annexins could establish initial protein-protein (or protein-phospholipid) interactions between LE/MVB and ER membranes to induce MCS formation and allow the exchange of ions and lipids, including cholesterol in other physiological settings (Miwa et al., 2008; Rintala-Dempsey et al., 2008). 
AnxA6 has recently also been associated with MCS formation (Meneses-Salas et al., 2020b). Further to its association with the plasma membrane, endo-/exocytic vesicles, mitochondria and lipid droplets (Grewal et al., 2021), AnxA6 was additionally detected in MAMs (Sala-Vila et al., 2016), specialized membrane subdomains enriched in cholesterol and neutral lipids that permit the communication between the ER and mitochondria (Vance, 1990; Hayashi and Su, 2003).

Given the recently identified role of AnxA6 in cholesterol transfer across MCS (Meneses-Salas et al., 2020b) and its presence in MAMs (Sala-Vila et al., 2016), the control of cholesterol transfer across MAMs and its alignment with steroid, oxysterol and bile acid synthesis is decisive for proper mitochondrial homeostasis. Emerging molecular insights include the identification of the founder member of the START [(Steroidogenic Acute Regulatory protein) related lipid transfer] domain family, StAR, also known as StARD1, at the outer mitochondrial membrane (OMM) as part of a multiprotein complex, with the voltage-dependent anion-selective channel protein (VDAC) and phosphate carrier protein (PCP), involved in the import of cholesterol (Reitz et al., 2008; Alpy and Tomasetto, 2014; Elustondo et al., 2017). StARD1 first incorporates ER-derived cholesterol into OMM, and together with VDAC and the translocator protein (TSPO), interacts with ATPase family AAA domain containing 3A (ATAD3A) and cytochrome P450 family 11 subfamily A member 1 (CYP11A1) in the inner mitochondrial membrane (Rone et al., 2012), to move and then metabolize cholesterol (Elustondo et al., 2017). Yet, how the feedback loop that coordinates LDL-derived cholesterol uptake and de novo cholesterol synthesis links to tethering and untethering events between the ER or LE/Lys with mitochondria to prevent excessive cholesterol transfer across MAMs remains unclear. Despite regulatory roles for AnxA6 in cholesterol transfer across LE/Lys-ER contacts (see below), similar functions for AnxA6 in MAMs have yet to be identified.

This indicates that mechanisms are in place that keep alternative and NPC1-independent cholesterol transport in an inactive state and do not enable other transport machinery to overcome cholesterol accumulation caused by NPC1 deficiency. Therefore, the presence of yet unidentified inhibitory proteins that act as "gatekeepers" may control activation of alternative cholesterol transport routes exiting LE/Lys. Indeed, NPC1 deficiency was associated with downregulation of the GTPase Rab7, the master regulator of LE/Lys function. Inhibition of Rab7 activity was mediated by AnxA6, which recruited the Rab7GTPase activating protein (Rab7-GAP) TBC1 domain family member 15 (TBC1D15) to cholesterol-rich LE, thereby lowering Rab7-GTP levels. Strikingly, AnxA6 depletion in NPC1 mutant cells and the concomitant loss of TBC1D15 membrane targeting elevated Rab7-GTP levels, leading to increased MCS formation between LE/Lys and the ER (Figure 1B2). This MCS restoration enabled cholesterol transfer across LE/Lys-ER contacts via the cholesterol transporter StARD3 for storage in lipid droplets. Hence, the AnxA6/TBC1D15 complex could become a potential therapeutic target to slow down the progressive neurodegeneration in NPC disease (Enrich et al., 2019; Meneses-Salas et al., 2020b).

Interestingly, loss of TBC1D15-mediated Rab7-GTP hydrolysis also inhibited the untethering of mitochondria-LE/ Lys contacts, disrupting mitochondrial distribution and function in models mimicking Parkinson's disease pathophysiology (Wong Y. C. et al., 2018; Kim et al., 2021) (Figure 1B3). Similarly, Rab7 mutations with reduced GTPase activity in Charcot-Marie-Tooth type 2B (CMT2B) are linked to defective mitochondria-lysosome contact dynamics (Bucci and De Luca, 2012; Wong et al., 2019). Of note, in mitochondria, AnxA6 also interacts with dynamin-related protein 1 (Drp1) (Chlystun et al., 2013), a GTPase interconnected with Rab7-dependent mitochondrial-LE/Lys contact formation in CMT2B (Wong et al., 2019). Hence, one can envisage a scaffolding role for AnxA6 in Rab7/TBC1D15 and Drp1-dependent dynamics of mitochondria-LE/Lys contacts in these neurological diseases (Figure 1B4).

Studies described above suggest annexin levels to differentially impact on MCS numbers, composition and function. Indeed, AnxA1 depletion markedly reduced MCS connecting EGFRcontaining MVBs and the ER, while MCS between EGFRdeficient MVBs and the ER remained unaffected (Eden et al., 2016). Likewise, MCS exist in NPC mutant cells, in particular between LE/Lys and mitochondria (Hoglinger et al., 2019), yet AnxA6 depletion and consequently, loss of TBC1D15 recruitment to LE/Lys in these cells, increased MCS numbers between LE/Lys and the ER for cholesterol transfer, requiring Rab7, and the cholesterol transporter StARD3. Hence, high/low annexin levels acting as tethers (AnxA1) or gatekeepers (AnxA6) will differentially influence MCS protein composition and functions, with consequences for cholesterol transport between organelles. This may extend to other annexins, including AnxA2, which together with S100A10, can bridge membranes (Illien et al., 2012; Grill et al., 2018; Berg Klenow et al., 2021), and bind to cholesterol-rich LE (Mayran et al., 2003). Similarly, AnxA8 is recruited to cholesterol-laden LE, and AnxA8 depletion caused LE/Lys cholesterol accumulation (Heitzig et al., 2018). Further examples of up- or downregulated tethers, with consequences for lipid- or ion-related MCS transfer, comprise phosphatase interacting protein 51 (PTPIP51, also called RMDN3) (Galmes et al., 2016), and PDZ domain-containing protein 8 (PDZD8) (Hirabayashi et al., 2017). Thus, manipulating the levels of tethers, untethers and lipid transporters (Galmes et al., 2016; Pulli et al., 2018) can offer therapeutic opportunities to modulate MCS formation in disease.

\section{Protein Domains in Annexins That Could Modulate MCS Assembly}

The potential involvement of annexins in MCS formation by means of interactions with FFAT motifs (two phenylalanines in an acidic tract) in MCS-associated proteins should also be considered. FFAT motifs were originally identified in late endosomal/lysosomal proteins, interacting with ER-associated VAPs. Several variations of the original FFAT motif exist (Mikitova and Levine, 2012; Murphy and Levine, 2016; 
Cabukusta et al., 2020), including the Phospho-FFAT motif in AnxA5 and A8 (Di Mattia et al., 2020). Homologies to FFAT motifs were also located in AnxA1, A6 and A11 (Figure 1C) (Rentero et al., 2018). Given their association with the LE/Lys compartment and affinity for cholesterol (de Diego et al., 2002; Hulce et al., 2013), one could envisage MCS formation between the ER and LE/Lys being influenced by ER-associated VAPs or motile sperm domain-containing proteins (MOSPDs) recognizing FFAT-like motifs in these annexins.

Alternatively, post-translational modifications such as palmitoylation, which enables the targeting of cytosolic proteins to membranes, often modulating the activity of multiprotein complexes in specialized microdomains (Charollais and Van Der Goot, 2009). This is exemplified by palmitoylated caveolin-1 and its ability to bind cholesterol, thereby determining the cholesterol content of ERmitochondria subdomains, linking organelle communication across MAMs with intracellular steroid and lipoprotein metabolism (Sala-Vila et al., 2016).

Likewise, palmitoylated cytoskeleton-associated protein 4 (CKAP4) interacts with VDAC2 at ER-mitochondrial contacts (Harada et al., 2020). This could impact on cholesterol transfer, as StARD1, which transfers ER-derived cholesterol to mitochondria, can form a complex with TOM22 and VDAC2 (see above) (Torres et al., 2017; Gordaliza-Alaguero et al., 2019).

Another example is the transmembrane protein $55 \mathrm{~B}$ (TMEM55B), which interacts with the cytosolic scaffold protein JIP4 and dynein/dynactin in MCS to modulate the spatial distribution and positioning of lysosomes. TMEM55B palmitoylation was decisive for lysosomal positioning, implicating a critical role in determining the speed and location of MCS being formed, (Ballabio and Bonifacino, 2020; Rudnik et al., 2021; Saftig and Puertollano, 2021).

Interestingly, AnxA1 and AnxA6 were recently identified as palmitoylated substrates in extracellular vesicle fractions, including exosomes (Albacete-Albacete et al., 2020; Mariscal et al., 2020). The mechanisms that regulate this posttranslational modification or whether this modification also applies for other annexins remains unknown. Hence, palmitoylated annexins may also contribute to MCS tethering. Exploring whether annexins establish palmitoylated links with perimeter LE/Lys membranes as well as mutational analysis of the FFAT-like motif in annexins will address the relevance and potential consequences of these proposed interactions.

\section{AnxA11 is a Tether of Lysosomes and RNA Granules}

Recently, AnxA11 was detected in lysosomes that connect with RNA granules (Liao et al., 2019). Alike AnxA1 and A6, AnxA11 is widely expressed with diverse, often $\mathrm{Ca}^{2+}$ - and S100A6 (calcyclin)-dependent functions in cytoplasmic and nuclear membrane locations, relevant for growth, cell cycle progression, differentiation, and exocytosis (Grewal et al., 2021).

Liao and coworkers identified AnxA11 as a tether mediating RNA granule association with lysosomes during their transport to distal regions of the axon (Liao et al., 2019). Strikingly, AnxA11 mutations associated with amyotrophic lateral sclerosis (ALS) disrupted docking between RNA granules and lysosomes, thus hampering neuronal RNA granule transport. Mutant analysis mapped the AnxA11 N-terminus as necessary for $\mathrm{Ca}^{2+}$ - and phospholipid-dependent lysosome-RNA granule interactions, which could be relevant for RNA granule microtubule-based transport in polarized epithelial cells or neurons, facilitating local protein translation at subcellular locations (Lee et al., 2020; Das et al., 2021). Hence, AnxA11 represents a novel mechanistic and structural link between lysosomes and a membraneless compartment in ALS pathogenesis. This observation might extend to other annexins, as the AnxA11 interactome included AnxA7 (Liao et al., 2019). Similarly, using AnxA6 as bait, we identified AnxA11 and AnxA7 as binding partners, indicating that interactions between multiple annexins, as proposed previously (Li et al., 2016), may contribute to the tethering of lysosomes to other organelles.

\section{DISCUSSION}

Since the discovery of the annexin domain structure (Geisow, 1986), annexins have been identified in many organisms, including humans (Moss and Morgan, 2004). The $\mathrm{Ca}^{2+}$-inducible conformational change and differential preference for negatively charged phospholipids and other lipids, in particular cholesterol, can enhance membrane association of several annexins (Enrich et al., 2017). Furthermore, their promiscuous behaviour to differentially interact with other proteins together with their innate properties to "annex" membranes, make annexins suitable applicants for MCS appointments.

\section{Annexins: Regulators of Cholesterol Trafficking, ILV Formation and MCS Association}

As outlined above, AnxA1 and AnxA6 control cholesterol transport from the ER to LE/Lys and vice versa via MCS (Eden et al., 2016; Meneses-Salas et al., 2020b). This contribution to MCS functioning may assist to control cholesterol levels in MVBs to participate, together with other lipids and accessory proteins (Gruenberg, 2020), in ILV biogenesis. This might even create transport specificity, as AnxA1 only mediates MCS formation between the ER and EGFR-containing MVBs (Wong L. H. et al., 2018).

However, annexin recruitment to MCS remains to be clarified. Besides the potential contribution of FFAT motifs or palmitoylation listed above, this could occur via translocation of cytosolic annexin pools where local $\mathrm{Ca}^{2+}$, cholesterol or annexin-binding proteins could contribute to their association with MCS. Alternatively, annexin pools inside ILVs could be released via back fusion, a constitutive process occurring in MVBs, where ILV fuse with the perimeter LE membrane leaving the cargo at cytosolic interfaces delimited by juxtaposed membranes of MVBs and other organelles (i.e., ER) (Gruenberg, 2020; Perrin et al., 2021). In this scenario, annexins could locally encounter a suitable $\mathrm{Ca}^{2+}$ and lipid microenvironment that would enable them to act as 
interorganelle tethers. On the other hand, annexins on ILVs facing the lumen of MVBs might be part of the fusion machinery (including LBPA, cholesterol, Alix) for the back fusion process. In fact, some of the complex protein networks that interact with AnxA6 are also involved in ILV formation and may regulate back fusion (Enrich et al., 2017). The well-documented presence of annexins in exosomes provides credibility for mechanisms such as chaperone-mediated autophagy (CMA) or endosome microautophagy (e-MI) for annexin association with ILV, the latter having the ability to keep proteins inside ILV. This is supported by all annexins harbouring the KFERQ motif (Figure 1C) (Cuervo et al., 2000; Kaushik and Cuervo, 2018), which is considered responsible for the location of annexins inside ILV (White et al., 2006; Meneses-Salas et al., 2020a).

Overall, the evidence of annexins contributing to MCS formation and function in LE/Lys is growing, with consequences for membrane traffic, microdomain organization, interactions with the cytoskeleton, cholesterol homeostasis, tethering, $\mathrm{Ca}^{2+}$ signalling and positioning of acidic compartments, and likely relevant for many biological settings.

\section{REFERENCES}

Abrisch, R. G., Gumbin, S. C., Wisniewski, B. T., Lackner, L. L., and Voeltz, G. K. (2020). Fission and Fusion Machineries Converge at ER Contact Sites to Regulate Mitochondrial Morphology. J. Cel Biol 219 (4), e201911122. doi:10.1083/jcb.201911122

Albacete-Albacete, L., Navarro-Lérida, I., López, J. A., Martín-Padura, I., Astudillo, A. M., Ferrarini, A., et al. (2020). ECM Deposition Is Driven by Caveolin-1dependent Regulation of Exosomal Biogenesis and Cargo Sorting. J. Cel Biol 219 (11), e202006178. doi:10.1083/jcb.202006178

Alpy, F., and Tomasetto, C. (2005). Give Lipids a START: the StAR-related Lipid Transfer (START) Domain in Mammals. J. Cel Sci 118 (Pt 13), 2791-2801. doi:10.1242/jcs.02485

Alpy, F., and Tomasetto, C. (2014). START Ships Lipids across Interorganelle Space. Biochimie 96, 85-95. doi:10.1016/j.biochi.2013.09.015

Alvarez-Guaita, A., Blanco-Muñoz, P., Meneses-Salas, E., Wahba, M., Pollock, A. H., Jose, J., et al. (2020). Annexin A6 Is Critical to Maintain Glucose Homeostasis and Survival during Liver Regeneration in Mice. Hepatology 72 (6), 2149-2164. doi:10.1002/hep.31232

Atakpa, P., Thillaiappan, N. B., Mataragka, S., Prole, D. L., and Taylor, C. W. (2018). IP3 Receptors Preferentially Associate with ER-Lysosome Contact Sites and Selectively Deliver Ca2+ to Lysosomes. Cel Rep. 25 (11), 3180-3193.e7. doi:10.1016/j.celrep.2018.11.064

Balla, T., Kim, Y. J., Alvarez-Prats, A., and Pemberton, J. (2019). Lipid Dynamics at Contact Sites between the Endoplasmic Reticulum and Other Organelles. Annu. Rev. Cel Dev. Biol. 35, 85-109. doi:10.1146/annurev-cellbio-100818-125251

Ballabio, A., and Bonifacino, J. S. (2020). Lysosomes as Dynamic Regulators of Cell and Organismal Homeostasis. Nat. Rev. Mol. Cel Biol 21 (2), 101-118. doi:10.1038/s41580-019-0185-4

Berg Klenow, M., Iversen, C., Wendelboe Lund, F., Mularski, A., Busk Heitmann, A. S., Dias, C., et al. (2021). Annexins A1 and A2 Accumulate and Are Immobilized at Cross-Linked Membrane-Membrane Interfaces. Biochemistry 60 (16), 1248-1259. doi:10.1021/acs.biochem.1c00126

Bohnert, M. (2019). Organelle Contact Sites: Lipid Droplets Hooked by Metabolically Controlled Tethers. Curr. Biol. 29 (10), R375-R377. doi:10.1016/j.cub.2019.03.049

Bucci, C., and De Luca, M. (2012). Molecular Basis of Charcot-Marie-Tooth Type 2B Disease. Biochem. Soc. Trans. 40 (6), 1368-1372. doi:10.1042/BST20120197

Cabukusta, B., Berlin, I., van Elsland, D. M., Forkink, I., Spits, M., de Jong, A. W. M., et al. (2020). Human VAPome Analysis Reveals MOSPD1 and

\section{AUTHOR CONTRIBUTIONS}

$\mathrm{AL}$, inputs and discussion FT, reading, criticisms and discussion $\mathrm{CR}$, art work and discussion TG, editing, discussion CE, write the manuscript and coordinate the review.

\section{FUNDING}

CR is supported by the Serra Húnter Programme (Generalitat de Catalunya). AL is supported by the Michael J Fox Foundation for Parkinson's Research. TG is supported by the University of Sydney, Sydney, Australia.

\section{ACKNOWLEDGMENTS}

$\mathrm{CE}$ and $\mathrm{CR}$ acknowledge the funding from Ministerio de Ciencia e Innovación (Spain, Grant PID 2020-115910RB-I00). CE is also thankful to the University of Barcelona (Grant AR0RM005) and UIU2019 fellowship.

MOSPD3 as Membrane Contact Site Proteins Interacting with FFATRelated FFNT Motifs. Cel Rep. 33 (10), 108475. doi:10.1016/ j.celrep.2020.108475

Chang, N., Sutherland, C., Hesse, E., Winkfein, R., Wiehler, W. B., Pho, M., et al. (2007). Identification of a Novel Interaction between the Ca2+-Binding Protein S100A11 and the Ca2+- and Phospholipid-Binding Protein Annexin A6. Am. J. Physiology-Cell Physiol. 292 (4), C1417-C1430. doi:10.1152/ ajpcell.00439.2006

Charollais, J., and Van Der Goot, F. G. (2009). Palmitoylation of Membrane Proteins (Review). Mol. Membr. Biol. 26 (1), 55-66. doi:10.1080/ 09687680802620369

Chlystun, M., Campanella, M., Law, A.-L., Duchen, M. R., Fatimathas, L., Levine, T. P., et al. (2013). Regulation of Mitochondrial Morphogenesis by Annexin A6. PLoS One 8 (1), e53774. doi:10.1371/journal.pone.0053774

Cremer, T., Neefjes, J., and Berlin, I. (2020). The Journey of Ca2+ through the Cell Pulsing through the Network of ER Membrane Contact Sites. J. Cel Sci 133 (24), jcs249136. doi:10.1242/jcs.249136

Cuervo, A. M., Gomes, A. V., Barnes, J. A., and Dice, J. F. (2000). Selective Degradation of Annexins by Chaperone-Mediated Autophagy. J. Biol. Chem. 275 (43), 33329-33335. doi:10.1074/jbc.M005655200

Das, S., Vera, M., Gandin, V., Singer, R. H., and Tutucci, E. (2021). Intracellular mRNA Transport and Localized Translation. Nat. Rev. Mol. Cel Biol 22 (7), 483-504. doi:10.1038/s41580-021-00356-8

de Diego, I., Schwartz, F., Siegfried, H., Dauterstedt, P., Heeren, J., Beisiegel, U., et al. (2002). Cholesterol Modulates the Membrane Binding and Intracellular Distribution of Annexin 6. J. Biol. Chem. 277 (35), 32187-32194. doi:10.1074/ jbc.M205499200

Di Mattia, T., Martinet, A., Ikhlef, S., McEwen, A. G., Nominé, Y., Wendling, C., et al. (2020). FFAT Motif Phosphorylation Controls Formation and Lipid Transfer Function of Inter-organelle Contacts. EMBO J. 39 (23), e104369. doi:10.15252/embj.2019104369

Dong, R., Saheki, Y., Swarup, S., Lucast, L., Harper, J. W., and De Camilli, P. (2016). Endosome-ER Contacts Control Actin Nucleation and Retromer Function through VAP-dependent Regulation of PI4P. Cell 166 (2), 408-423. doi:10.1016/j.cell.2016.06.037

Du, X., Kazim, A. S., Brown, A. J., and Yang, H. (2012). An Essential Role of Hrs/ Vps27 in Endosomal Cholesterol Trafficking. Cel Rep. 1 (1), 29-35. doi:10.1016/ j.celrep.2011.10.004

Eden, E. R., and Futter, C. E. (2021). Membrane Trafficking: Retrofusion as an Escape Route Out of the Endosome. Curr. Biol. 31 (17), R1037-R1040. doi:10.1016/j.cub.2021.07.055 
Eden, E. R., Sanchez-Heras, E., Tsapara, A., Sobota, A., Levine, T. P., and Futter, C. E. (2016). Annexin A1 Tethers Membrane Contact Sites that Mediate ER to Endosome Cholesterol Transport. Develop. Cel 37 (5), 473-483. doi:10.1016/ j.devcel.2016.05.005

Eden, E. R., White, I. J., Tsapara, A., and Futter, C. E. (2010). Membrane Contacts between Endosomes and ER Provide Sites for PTP1B-Epidermal Growth Factor Receptor Interaction. Nat. Cel Biol 12 (3), 267-272. doi:10.1038/ncb2026

Eisenberg-Bord, M., Shai, N., Schuldiner, M., and Bohnert, M. (2016). A Tether Is a Tether Is a Tether: Tethering at Membrane Contact Sites. Develop. Cel 39 (4), 395-409. doi:10.1016/j.devcel.2016.10.022

Elustondo, P., Martin, L. A., and Karten, B. (2017). Mitochondrial Cholesterol Import. Biochim. Biophys. Acta (Bba) - Mol. Cel Biol. Lipids 1862 (1), 90-101. doi:10.1016/j.bbalip.2016.08.012

Enrich, C., Rentero, C., Grewal, T., Futter, C. E., and Eden, E. R. (2019). Cholesterol Overload: Contact Sites to the Rescue!. Contact 2, 251525641989350. doi:10.1177/2515256419893507

Enrich, C., Rentero, C., Meneses-Salas, E., Tebar, F., and Grewal, T. (2017). Annexins: Ca2+ Effectors Determining Membrane Trafficking in the Late Endocytic Compartment. Adv. Exp. Med. Biol. 981, 351-385. doi:10.1007/ 978-3-319-55858-5_14

Friedman, J. R., Dibenedetto, J. R., West, M., Rowland, A. A., and Voeltz, G. K. (2013). Endoplasmic Reticulum-Endosome Contact Increases as Endosomes Traffic and Mature. MBoC 24 (7), 1030-1040. doi:10.1091/mbc.E12-10-0733

Futter, C. E., Felder, S., Schlessinger, J., Ullrich, A., and Hopkins, C. R. (1993). Annexin I Is Phosphorylated in the Multivesicular Body during the Processing of the Epidermal Growth Factor Receptor. J. Cel Biol 120 (1), 77-83. doi:10.1083/jcb.120.1.77

Galmes, R., Houcine, A., Vliet, A. R., Agostinis, P., Jackson, C. L., and Giordano, F. (2016). ORP5/ORP8 Localize to Endoplasmic Reticulum-Mitochondria Contacts and Are Involved in Mitochondrial Function. EMBO Rep. 17 (6), 800-810. doi:10.15252/embr.201541108

Geisow, M. J. (1986). Common Domain Structure of Ca2+and Lipid-Binding Proteins. FEBS Lett. 203 (1), 99-103. doi:10.1016/0014-5793(86)81445-4

Gerke, V., Creutz, C. E., and Moss, S. E. (2005). Annexins: Linking Ca2+ Signalling to Membrane Dynamics. Nat. Rev. Mol. Cel Biol 6 (6), 449-461. doi:10.1038/ nrm1661

Gordaliza-Alaguero, I., Cantó, C., and Zorzano, A. (2019). Metabolic Implications of Organelle-Mitochondria Communication. EMBO Rep. 20 (9), e47928. doi:10.15252/embr.201947928

Grewal, T., Rentero, C., Enrich, C., Wahba, M., Raabe, C. A., and Rescher, U. (2021). Annexin Animal Models-From Fundamental Principles to Translational Research. Int. J. Mol. Sci. 22 (7), 3439. doi:10.3390/ijms22073439

Grill, D., Matos, A. L. L., de Vries, W. C., Kudruk, S., Heflik, M., Dörner, W., et al. (2018). Bridging of Membrane Surfaces by Annexin A2. Sci. Rep. 8 (1), 14662. doi:10.1038/s41598-018-33044-3

Gruenberg, J. (2020). Life in the Lumen: The Multivesicular Endosome. Traffic 21 (1), 76-93. doi:10.1111/tra.12715

Harada, T., Sada, R., Osugi, Y., Matsumoto, S., Matsuda, T., Hayashi-Nishino, M., et al. (2020). Palmitoylated CKAP4 Regulates Mitochondrial Functions through an Interaction with VDAC2 at ER-Mitochondria Contact Sites. J. Cel Sci 133 (21), jcs249045. doi:10.1242/jcs. 249045

Hayashi, T., and Su, T.-P. (2003). $\sigma$-1 Receptors ( $\sigma 1$ Binding Sites) Form Raft-like Microdomains and Target Lipid Droplets on the Endoplasmic Reticulum: Roles in Endoplasmic Reticulum Lipid Compartmentalization and Export. J. Pharmacol. Exp. Ther. 306 (2), 718-725. doi:10.1124/jpet.103.051284

Heitzig, N., Kühnl, A., Grill, D., Ludewig, K., Schloer, S., Galla, H.-J., et al. (2018). Cooperative Binding Promotes Demand-Driven Recruitment of AnxA8 to Cholesterol-Containing Membranes. Biochim. Biophys. Acta (Bba) - Mol. Cel Biol. Lipids 1863 (4), 349-358. doi:10.1016/j.bbalip.2018.01.001

Henne, M. (2019). And Three's a Party: Lysosomes, Lipid Droplets, and the ER in Lipid Trafficking and Cell Homeostasis. Curr. Opin. Cel Biol. 59, 40-49. doi:10.1016/j.ceb.2019.02.011

Henne, W. M., Zhu, L., Balogi, Z., Stefan, C., Pleiss, J. A., and Emr, S. D. (2015). $\mathrm{Mdm} 1 / \mathrm{Snx} 13$ Is a Novel ER-Endolysosomal Interorganelle Tethering Protein. J. Cel Biol 210 (4), 541-551. doi:10.1083/jcb.201503088

Hewlett, B., Singh, N. P., Vannier, C., and Galli, T. (2021). ER-PM Contact Sites SNARING Actors in Emerging Functions. Front. Cel Dev. Biol. 9, 635518. doi:10.3389/fcell.2021.635518
Hirabayashi, Y., Kwon, S.-K., Paek, H., Pernice, W. M., Paul, M. A., Lee, J., et al. (2017). ER-mitochondria Tethering by PDZD8 Regulates Ca2+dynamics in Mammalian Neurons. Science 358 (6363), 623-630. doi:10.1126/ science.aan6009

Höglinger, D., Burgoyne, T., Sanchez-Heras, E., Hartwig, P., Colaco, A., Newton, J., et al. (2019). NPC1 Regulates ER Contacts with Endocytic Organelles to Mediate Cholesterol Egress. Nat. Commun. 10 (1), 4276. doi:10.1038/ s41467-019-12152-2

Hoyer, M. J., Chitwood, P. J., Ebmeier, C. C., Striepen, J. F., Qi, R. Z., Old, W. M., et al. (2018). A Novel Class of ER Membrane Proteins Regulates ER-Associated Endosome Fission. Cell 175 (1), 254-265.e14. doi:10.1016/j.cell.2018.08.030

Hulce, J. J., Cognetta, A. B., Niphakis, M. J., Tully, S. E., and Cravatt, B. F. (2013). Proteome-wide Mapping of Cholesterol-Interacting Proteins in Mammalian Cells. Nat. Methods 10 (3), 259-264. doi:10.1038/nmeth.2368

Illien, F., Piao, H.-R., Coué, M., di Marco, C., and Ayala-Sanmartin, J. (2012). Lipid Organization Regulates Annexin A2 Ca2+-Sensitivity for Membrane Bridging and its Modulator Effects on Membrane Fluidity. Biochim. Biophys. Acta (Bba) Biomembranes 1818 (11), 2892-2900. doi:10.1016/j.bbamem.2012.07.012

Islinger, M., Costello, J. L., Kors, S., Soupene, E., Levine, T. P., Kuypers, F. A., et al. (2020). The Diversity of ACBD Proteins - from Lipid Binding to Protein Modulators and Organelle Tethers. Biochim. Biophys. Acta (Bba) - Mol. Cel Res. 1867 (5), 118675. doi:10.1016/j.bbamcr.2020.118675

Kaushik, S., and Cuervo, A. M. (2018). The Coming of Age of Chaperone-Mediated Autophagy. Nat. Rev. Mol. Cel Biol 19 (6), 365-381. doi:10.1038/s41580-0180001-6

Kentala, H., Weber-Boyvat, M., and Olkkonen, V. M. (2016). OSBP-related Protein Family: Mediators of Lipid Transport and Signaling at Membrane Contact Sites. Int. Rev. Cel Mol Biol 321, 299-340. doi:10.1016/bs.ircmb.2015.09.006

Kim, S., Wong, Y. C., Gao, F., and Krainc, D. (2021). Dysregulation of Mitochondria-Lysosome Contacts by GBA1 Dysfunction in Dopaminergic Neuronal Models of Parkinson's Disease. Nat. Commun. 12 (1), 1807. doi:10.1038/s41467-021-22113-3

Kumar, N., Leonzino, M., Hancock-Cerutti, W., Horenkamp, F. A., Li, P., Lees, J. A., et al. (2018). VPS13A and VPS13C Are Lipid Transport Proteins Differentially Localized at ER Contact Sites. J. Cel Biol 217 (10), 3625-3639. doi:10.1083/jcb.201807019

Lee, J. E., Cathey, P. I., Wu, H., Parker, R., and Voeltz, G. K. (2020). Endoplasmic Reticulum Contact Sites Regulate the Dynamics of Membraneless Organelles. Science 367 (6477), eaay7108. doi:10.1126/science.aay7108

Li, H.-D., Menon, R., Eksi, R., Guerler, A., Zhang, Y., Omenn, G. S., et al. (2016). A Network of Splice Isoforms for the Mouse. Sci. Rep. 6, 24507. doi:10.1038/ srep24507

Liao, Y.-C., Fernandopulle, M. S., Wang, G., Choi, H., Hao, L., Drerup, C. M., et al. (2019). RNA Granules Hitchhike on Lysosomes for Long-Distance Transport, Using Annexin A11 as a Molecular Tether. Cell 179 (1), 147-164. doi:10.1016/ j.cell.2019.08.050

Mariscal, J., Vagner, T., Kim, M., Zhou, B., Chin, A., Zandian, M., et al. (2020). Comprehensive Palmitoyl-proteomic Analysis Identifies Distinct Protein Signatures for Large and Small Cancer-derived Extracellular Vesicles. J. Extracellular Vesicles 9 (1), 1764192. doi:10.1080/ 20013078.2020.1764192

Martello, A., Platt, F. M., and Eden, E. R. (2020). Staying in Touch with the Endocytic Network: The Importance of Contacts for Cholesterol Transport. Traffic 21 (5), 354-363. doi:10.1111/tra.12726

Mayran, N., Parton, R. G., and Gruenberg, J. (2003). Annexin II Regulates Multivesicular Endosome Biogenesis in the Degradation Pathway of Animal Cells. EMBO J. 22 (13), 3242-3253. doi:10.1093/emboj/cdg321

Meneses-Salas, E., García-Melero, A., Blanco-Muñoz, P., Jose, J., Brenner, M.-S., Lu, A., et al. (2020a). Selective Degradation Permits a Feedback Loop Controlling Annexin A6 and Cholesterol Levels in Endolysosomes of NPC1 Mutant Cells. Cells 9 (5), 1152. doi:10.3390/cells9051152

Meneses-Salas, E., García-Melero, A., Kanerva, K., Blanco-Muñoz, P., Morales-Paytuvi, F., Bonjoch, J., et al. (2020b). Annexin A6 Modulates TBC1D15/Rab7/StARD3 axis to Control Endosomal Cholesterol export in NPC1 Cells. Cell. Mol. Life Sci. 77 (14), 2839-2857. doi:10.1007/s00018019-03330-y

Mikitova, V., and Levine, T. P. (2012). Analysis of the Key Elements of FFAT-like Motifs Identifies New Proteins that Potentially Bind VAP on the ER, Including 
Two AKAPs and FAPP2. PLoS One 7 (1), e30455. doi:10.1371/ journal.pone. 0030455

Miwa, N., Uebi, T., and Kawamura, S. (2008). S100-annexin Complexes - Biology of Conditional Association. FEBS J. 275 (20), 4945-4955. doi:10.1111/j.17424658.2008.06653.x

Moss, S. E., and Morgan, R. O. (2004). The Annexins. Genome Biol. 5 (4), 219. doi:10.1186/gb-2004-5-4-219

Murphy, S. E., and Levine, T. P. (2016). VAP, a Versatile Access Point for the Endoplasmic Reticulum: Review and Analysis of FFAT-like Motifs in the VAPome. Biochim. Biophys. Acta (Bba) - Mol. Cel Biol. Lipids 1861 (8 Pt B), 952-961. doi:10.1016/j.bbalip.2016.02.009

Neefjes, J., and Cabukusta, B. (2021). What the VAP: The Expanded VAP Family of Proteins Interacting with FFAT and FFAT-Related Motifs for Interorganellar Contact. Contact 4, 251525642110122. doi:10.1177/ 25152564211012246

Nixon-Abell, J., Obara, C. J., Weigel, A. V., Li, D., Legant, W. R., Xu, C. S., et al. (2016). Increased Spatiotemporal Resolution Reveals Highly Dynamic Dense Tubular Matrices in the Peripheral ER. Science 354 (6311), aaf3928. doi:10.1126/science.aaf3928

Patel, S. (2019). Getting Close. Lysosome-ER Contact Sites Tailor Ca2+ Signals. Cell Calcium 80, 194-196. doi:10.1016/j.ceca.2019.02.003

Peng, W., Wong, Y. C., and Krainc, D. (2020). Mitochondria-lysosome Contacts Regulate Mitochondrial Ca2+dynamics via Lysosomal TRPML1. Proc. Natl. Acad. Sci. USA 117 (32), 19266-19275. doi:10.1073/pnas.2003236117

Perrin, P., Janssen, L., Janssen, H., van den Broek, B., Voortman, L. M., van Elsland, D., et al. (2021). Retrofusion of Intralumenal MVB Membranes Parallels Viral Infection and Coexists with Exosome Release. Curr. Biol. 31 (17), 3884-3893.e4. doi:10.1016/j.cub.2021.06.022

Petkovic, M., O'Brien, C. E., and Jan, Y. N. (2021). Interorganelle Communication, Aging, and Neurodegeneration. Genes Dev. 35 (7-8), 449-469. doi:10.1101/ gad.346759.120

Pons, M., Ihrke, G., Koch, S., Biermer, M., Pol, A., Grewal, T., et al. (2000). Late Endocytic Compartments Are Major Sites of Annexin VI Localization in NRK Fibroblasts and Polarized WIF-B Hepatoma Cells. Exp. Cel Res. 257 (1), 33-47. doi:10.1006/excr.2000.4861

Prinz, W. A., Toulmay, A., and Balla, T. (2020). The Functional Universe of Membrane Contact Sites. Nat. Rev. Mol. Cel Biol 21 (1), 7-24. doi:10.1038/ s41580-019-0180-9

Pulli, I., Lassila, T., Pan, G., Yan, D., Olkkonen, V. M., and Törnquist, K. (2018). Oxysterol-binding Protein Related-Proteins (ORPs) 5 and 8 Regulate Calcium Signaling at Specific Cell Compartments. Cell Calcium 72, 62-69. doi:10.1016/ j.ceca.2018.03.001

Quon, E., Sere, Y. Y., Chauhan, N., Johansen, J., Sullivan, D. P., Dittman, J. S., et al. (2018). Endoplasmic Reticulum-Plasma Membrane Contact Sites Integrate Sterol and Phospholipid Regulation. Plos Biol. 16 (5), e2003864. doi:10.1371/journal.pbio.2003864

Raiborg, C., and Stenmark, H. (2009). The ESCRT Machinery in Endosomal Sorting of Ubiquitylated Membrane Proteins. Nature 458 (7237), 445-452. doi:10.1038/nature07961

Raiborg, C., Wenzel, E. M., Pedersen, N. M., Olsvik, H., Schink, K. O., Schultz, S. W., et al. (2015a). Repeated ER-Endosome Contacts Promote Endosome Translocation and Neurite Outgrowth. Nature 520 (7546), 234-238. doi:10.1038/nature14359

Raiborg, C., Wenzel, E. M., and Stenmark, H. (2015b). ER -endosome Contact Sites: Molecular Compositions and Functions. EMBO J. 34 (14), 1848-1858. doi:10.15252/embj.201591481

Reinisch, K. M., and Prinz, W. A. (2021). Mechanisms of Nonvesicular Lipid Transport. J. Cel Biol 220 (3), e202012058. doi:10.1083/jcb.202012058

Reitz, J., Gehrig-Burger, K., Strauss, J. F., 3rd, and Gimpl, G. (2008). Cholesterol Interaction with the Related Steroidogenic Acute Regulatory Lipid-Transfer (START) Domains of StAR (STARD1) and MLN64 (STARD3). FEBS J. 275 (8), 1790-1802. doi:10.1111/j.1742-4658.2008.06337.x

Rentero, C., Blanco-Muñoz, P., Meneses-Salas, E., Grewal, T., and Enrich, C. (2018). Annexins-Coordinators of Cholesterol Homeostasis in Endocytic Pathways. Int. J. Mol. Sci. 19 (5), 1444. doi:10.3390/ijms19051444
Rescher, U., and Gerke, V. (2004). Annexins - Unique Membrane Binding Proteins with Diverse Functions. J. Cel Sci 117 (Pt 13), 2631-2639. doi:10.1242/jcs.01245

Réty, S., Osterloh, D., Arié, J.-P., Tabaries, S., Seeman, J., Russo-Marie, F., et al. (2000). Structural Basis of the Ca2+-dependent Association between S100C (S100A11) and its Target, the N-Terminal Part of Annexin I. Structure 8 (2), 175-184. doi:10.1016/s0969-2126(00)00093-9

Rintala-Dempsey, A. C., Rezvanpour, A., and Shaw, G. S. (2008). S100-annexin Complexes - Structural Insights. FEBS J. 275 (20), 4956-4966. doi:10.1111/ j.1742-4658.2008.06654.x

Rone, M. B., Midzak, A. S., Issop, L., Rammouz, G., Jagannathan, S., Fan, J., et al. (2012). Identification of a Dynamic Mitochondrial Protein Complex Driving Cholesterol Import, Trafficking, and Metabolism to Steroid Hormones. Mol. Endocrinol. 26 (11), 1868-1882. doi:10.1210/me.20121159

Rudnik, S., Heybrock, S., Saftig, P., and Damme, M. (2021). S-palmitoylation Determines TMEM55B-dependent Positioning of Lysosomes. J. Cel Sci 135, jcs258566. doi:10.1242/jcs.258566

Saftig, P., and Puertollano, R. (2021). How Lysosomes Sense, Integrate, and Cope with Stress. Trends Biochem. Sci. 46 (2), 97-112. doi:10.1016/ j.tibs.2020.09.004

Sala-Vila, A., Navarro-Lérida, I., Sánchez-Alvarez, M., Bosch, M., Calvo, C., López, J. A., et al. (2016). Interplay between Hepatic Mitochondria-Associated Membranes, Lipid Metabolism and Caveolin-1 in Mice. Sci. Rep. 6, 27351. doi:10.1038/srep27351

Sandhu, J., Li, S., Fairall, L., Pfisterer, S. G., Gurnett, J. E., Xiao, X., et al. (2018). Aster Proteins Facilitate Nonvesicular Plasma Membrane to ER Cholesterol Transport in Mammalian Cells. Cell 175 (2), 514-529. doi:10.1016/ j.cell.2018.08.033

Saric, A., Freeman, S. A., Williamson, C. D., Jarnik, M., Guardia, C. M., Fernandopulle, M. S., et al. (2021). SNX19 Restricts Endolysosome Motility through Contacts with the Endoplasmic Reticulum. Nat. Commun. 12 (1), 4552. doi:10.1038/s41467-021-24709-1

Scorrano, L., De Matteis, M. A., Emr, S., Giordano, F., Hajnóczky, G., Kornmann, B., et al. (2019). Coming Together to Define Membrane Contact Sites. Nat. Commun. 10 (1), 1287. doi:10.1038/s41467-019-09253-3

Shirane, M., Wada, M., Morita, K., Hayashi, N., Kunimatsu, R., Matsumoto, Y., et al. (2020). Protrudin and PDZD8 Contribute to Neuronal Integrity by Promoting Lipid Extraction Required for Endosome Maturation. Nat. Commun. 11 (1), 4576. doi:10.1038/s41467-020-18413-9

Silva, B. S. C., DiGiovanni, L., Kumar, R., Carmichael, R. E., Kim, P. K., and Schrader, M. (2020). Maintaining Social Contacts: The Physiological Relevance of Organelle Interactions. Biochim. Biophys. Acta (Bba) - Mol. Cel Res. 1867 (11), 118800. doi:10.1016/j.bbamcr.2020.118800

Tekirdag, K., and Cuervo, A. M. (2018). Chaperone-mediated Autophagy and Endosomal Microautophagy: Jointed by a Chaperone. J. Biol. Chem. 293 (15), 5414-5424. doi:10.1074/jbc.R117.818237

Torres, S., Balboa, E., Zanlungo, S., Enrich, C., Garcia-Ruiz, C., and FernandezCheca, J. C. (2017). Lysosomal and Mitochondrial Liaisons in Niemann-Pick Disease. Front. Physiol. 8, 982. doi:10.3389/fphys.2017.00982

van der Kant, R., and Neefjes, J. (2014). Small Regulators, Major Consequences $\mathrm{Ca} 2+$ and Cholesterol at the Endosome-ER Interface. J. Cel Sci 127 (Pt 5), 929-938. doi:10.1242/jcs.137539

Vance, J. E. (1990). Phospholipid Synthesis in a Membrane Fraction Associated with Mitochondria. J. Biol. Chem. 265 (13), 7248-7256. doi:10.1016/s00219258(19)39106-9

White, I. J., Bailey, L. M., Aghakhani, M. R., Moss, S. E., and Futter, C. E. (2006). EGF Stimulates Annexin 1-dependent Inward Vesiculation in a Multivesicular Endosome Subpopulation. EMBO J. 25 (1), 1-12. doi:10.1038/sj.emboj.7600759

Wong, L. H., Eden, E. R., and Futter, C. E. (2018). Roles for ER:endosome Membrane Contact Sites in Ligand-Stimulated Intraluminal Vesicle Formation. Biochem. Soc. Trans. 46 (5), 1055-1062. doi:10.1042/BST20170432

Wong, Y. C., Peng, W., and Krainc, D. (2019). Lysosomal Regulation of Intermitochondrial Contact Fate and Motility in Charcot-Marie-Tooth Type 2. Develop. Cel 50 (3), 339-354.e4. doi:10.1016/j.devcel.2019.05.033 
Wong, Y. C., Ysselstein, D., and Krainc, D. (2018). Mitochondria-lysosome Contacts Regulate Mitochondrial Fission via RAB7 GTP Hydrolysis. Nature 554 (7692), 382-386. doi:10.1038/nature25486

Wu, H., Carvalho, P., and Voeltz, G. K. (2018). Here, There, and Everywhere: The Importance of ER Membrane Contact Sites. Science 361 (6401), eaan5835. doi:10.1126/science.aan5835

Wu, H., and Voeltz, G. K. (2021). Reticulon-3 Promotes Endosome Maturation at ER Membrane Contact Sites. Develop. Cel 56 (1), 52-66.e7. doi:10.1016/ j.devcel.2020.12.014

Conflict of Interest: The authors declare that the research was conducted in the absence of any commercial or financial relationships that could be construed as a potential conflict of interest.
Publisher's Note: All claims expressed in this article are solely those of the authors and do not necessarily represent those of their affiliated organizations, or those of the publisher, the editors and the reviewers. Any product that may be evaluated in this article, or claim that may be made by its manufacturer, is not guaranteed or endorsed by the publisher.

Copyright (C) 2022 Enrich, Lu, Tebar, Rentero and Grewal. This is an open-access article distributed under the terms of the Creative Commons Attribution License (CC $B Y)$. The use, distribution or reproduction in other forums is permitted, provided the original author(s) and the copyright owner(s) are credited and that the original publication in this journal is cited, in accordance with accepted academic practice. No use, distribution or reproduction is permitted which does not comply with these terms. 